\title{
Recent advances in optical diagnosis of oral cancers: review and future perspectives
}

Ola Ibrahim

Technological University Dublin

Hugh Byrne

Technological University Dublin, hugh.byrne@tudublin.ie

Fiona Lyng

Technological University Dublin, fiona.lyng@tudublin.ie

See next page for additional authors

Follow this and additional works at: https://arrow.tudublin.ie/biophonart

Part of the Analytical, Diagnostic and Therapeutic Techniques and Equipment Commons, and the Biological and Chemical Physics Commons

\section{Recommended Citation}

Lyng, F. et al. (2016) Recent advances in optical diagnosis of oral cancers: review and future perspectives", Head and Neck-Journal for the Sciences and Specialities of the Head and Neck, 38, E2403-E2411 (2016). doi:10.1002/hed.24293

This Article is brought to you for free and open access by the DIT Biophotonics and Imaging at ARROW@TU Dublin. It has been accepted for inclusion in Articles by an authorized administrator of ARROW@TU Dublin. For more information, please contact arrow.admin@tudublin.ie, aisling.coyne@tudublin.ie,gerard.connolly@tudublin.ie.

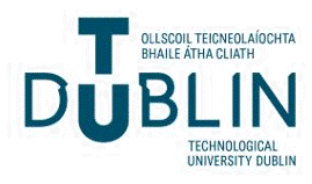


Authors

Ola Ibrahim, Hugh Byrne, Fiona Lyng, Surya Singh, Jopi Mikkonen, Arto Koistinen, and Arja Kullaa

This article is available at ARROW@TU Dublin: https://arrow.tudublin.ie/biophonart/26 


\section{Recent advances in optical diagnosis of oral cancers: review and future perspectives}

Surya P. Singh, $\mathrm{PhD}^{1,2}$, Ola Ibrahim, $\mathrm{MSc}^{3,4}$, Hugh J. Byrne, $\mathrm{PhD}^{5}$, Jopi W. Mikkonen, $\mathrm{MSc}^{2}$, Arto P. Koistinen, $\mathrm{PhD}^{2}$, Arja M. Kullaa, $\mathrm{PhD}^{1,6}$, Fiona M. Lyng, $\mathrm{PhD}^{3,4^{*}}$

${ }^{1}$ Institute of Dentistry, University of Eastern Finland, Kuopio, Finland

${ }^{2}$ SIB labs, University of Eastern Finland, Kuopio, Finland

${ }^{3}$ DIT Centre for Radiation and Environmental Science, FOCAS Research Institute, Dublin Institute of Technology, Ireland

${ }^{4}$ School of Physics, Dublin Institute of Technology, Ireland

${ }^{5}$ FOCAS Research Institute, Dublin Institute of Technology, Ireland

${ }^{6}$ Research Group of Oral Health Sciences, Faculty of Medicine, University of Oulu, Oulu, Finland

*Corresponding author:

Prof. Fiona M. Lyng

DIT Centre for Radiation and Environmental Science, FOCAS Research Institute, Dublin Institute of Technology, Kevin St, Dublin 8, Ireland

t: +35314027972

f: +35314027904

e : fiona.lyng@dit.ie

Running title: Optical diagnosis of oral cancers

Keywords: Raman spectroscopy, Infrared spectroscopy, Fluorescence spectroscopy, optical diagnosis, oral cancer, oral dysplasia

\section{Abstract}


Optical diagnosis techniques offer several advantages over traditional approaches, including objectivity, speed and cost, and these label-free, non-invasive methods have the potential to change the future work-flow of cancer management. The oral cavity is particularly accessible and thus such methods may serve as alternate/adjunct tools to traditional methods. Recently, in vivo human clinical studies have been initiated with a view to clinical translation of such technologies. A comprehensive review of optical methods in oral cancer diagnosis is presented. Following an introduction to the epidemiology and aetiological factors associated with oral cancers currently employed diagnostic methods and their limitations are presented. A thorough review of fluorescence, infrared absorption and Raman spectroscopic methods in oral cancer diagnosis is presented. The applicability of minimally invasive methods based on serum/saliva is also discussed. The review concludes with a discussion on future demands and scope of developments from a clinical point of view. 


\section{Oral Cancers: An Overview}

Oral squamous cell carcinoma (OSCC) ranks as the $15^{\text {th }}$ most common cancer in the world and $10^{\text {th }}$ most frequent in males. It accounts for $\sim 2.1 \%$ of total cancer cases worldwide. Incidence rates are high among males in south central Asia and among females in eastern and central Europe ${ }^{1}$. Although mortality from oral cancer has decreased in the past few decades, it is still high and has a five year survival rate of $50 \%^{2}$. Several oral lesions and conditions are associated with an increased potential for malignant transformation. Of these, the most commonly occurring is leukoplakia, defined as 'a predominantly white lesion of the oral mucosa that cannot be characterized as any other definable lesion' (Figure 1B). A wide range of malignant transformation rates have been reported, from 0.13$36.43 \%$, depending on the presence and degree of dysplasia, location in the oral cavity and maturity of the lesion ${ }^{3,4}$. Conversely, erythroplakia, defined as 'a fiery red patch which cannot be characterized clinically or pathologically as any other definable lesion, ${ }^{3}$, is less prevalent and has a higher potential for malignant transformation $(14.35-66.76 \%)^{5}$. Oral submucous fibrosis is a premalignant oral condition arising mostly due to areca nut or betel quid chewing ${ }^{6}$. It has a reported transformation rate of $7.6 \%{ }^{6}$. Oral lichen planus is an inflammatory disease of the oral mucosa. Like leukoplakia, it presents as a white plaque or patch. However, its malignant transformation rate is much lower than leukoplakia, $\sim 1 \%{ }^{7}$ (Figure 1C).

A number of risk factors are associated with oral cancer, including cigarette smoking and alcohol consumption, which are responsible for $42 \%$ and $16 \%$ of oral cancer deaths respectively ${ }^{8}$. The carcinogenicity of cigarette smoke has long been established, resulting in DNA damage and increase in P53 mutations ${ }^{9}$. The association between alcohol and oral cancer was reported to be dose dependent and a number of factors may contribute to its carcinogenicity, including acetaldehyde, an alcohol metabolite thought to be carcinogenic ${ }^{10}$. Moreover, alcohol is thought to act as a solvent for other carcinogens ${ }^{10}$. Oral habits such as smokeless tobacco and betel quid chewing are also implicated in oral cancer development ${ }^{11}$. They are more common in the Asian population; in India, $50 \%$ of oral cancers arise due to smokeless tobacco chewing ${ }^{11}$. The carcinogenicity of smokeless tobacco arises from the production of nitrosamines ${ }^{11}$. Oral squamous cell carcinoma has also been associated with 
the Epstein Bar (EBV) and Human Papilloma (HPV) Viruses, although their putative role is controversial. Around 23\% of OSCC were found to be positive for high risk HPV 16 and $18^{12}$.

\section{Current Screening/Diagnostic methods and limitations:}

Screening tests or diagnostic aids presently available for oral cancer include conventional oral examination (COE), staining with toluidine blue, oral brush biopsy and scalpel biopsy coupled with histology.

Conventional Oral Examination (COE): Conventional oral examination (COE), using normal (incandescent) light, has long been the standard screening method for oral abnormalities. As it is a visual method, it cannot identify early mucosal abnormalities that may or may not lead to oral cancer. Approximately 5-15\% of the general population has oral mucosal abnormalities and the vast majority of these lesions are benign in nature ${ }^{13}$, Furthermore, only a small percentage of leukoplakias are progressive or become malignant and COE cannot discriminate between these and their nonprogressive counterparts. Therefore, while COE may be useful in discovering some oral lesions, its potential in identifying all potentially premalignant or biologically relevant lesions that are likely to progress to cancer is questionable.

Toluidine blue staining: Toluidine blue (TB), has been used for more than 40 years as an aid to detection of mucosal abnormalities of the cervix and the oral cavity. TB is a metachromatic, acidophilic dye that binds preferentially to tissues undergoing rapid cell division (inflammatory, regenerative and neoplastic tissue), resulting in preferential staining of abnormal tissue. Overall, TB appears to be useful in detecting carcinomas, but is positive in only $\sim 50 \%$ of lesions with dysplasia. In addition, it frequently stains common, benign conditions such as non-specific ulcers. The high rate of false positive stains and the low specificity in staining dysplasia are some of the accepted limitations of the technique ${ }^{14}$.

Oral brush biopsy: Oral brush biopsy extracts a complete trans-epithelial biopsy specimen, with cellular representation from each of the three layers (basal, intermediate and superficial). Because the brush biopsy detects only cellular atypia, positive results must be confirmed with a scalpel biopsy for definitive diagnosis. This technique has therefore been criticized for adding time and cost to the 
diagnosis of oral lesions without additional benefit to the patient ${ }^{15}$. Overall, it is a method of identifying unsuspected oral cancers found during a visual examination, at early and curable stages ${ }^{16}$.

Histology: Histological risk stratification, currently the gold standard for oral cancer diagnosis, requires biopsy, staining and microscopic examination by a pathologist. However, removal of tissue or biopsy is an inherently invasive procedure and carries risk of complications in the proximity of vital anatomy. Sampling errors in collecting or interpreting biopsies due to inter-observer discrepancy can be significant. Once removed, the tissue can undergo biochemical changes which can lead to artifacts. In many diseases, tissue involvement is not uniform, potentially leading to sampling errors. Especially in oral cancers, some early lesions are clinically indistinguishable from benign conditions. Furthermore, histologically, identification of subtle changes in precancerous lesions or in normal mucosa that are indicative of early neoplastic transformation is subjective and can lead to interobserver variations ${ }^{17}$.

It is therefore conceivable that the primary prevention of the disease would involve activities to reduce or eliminate the use of tobacco and alcohol. Secondary prevention includes activities that are aimed to detect the disease at an early stage which would lead to better prognosis and lower morbidity. Current methods of detection of oral cancers are based largely on visual observations of abnormalities in tissue or cellular morphology and are therefore limited in terms of sensitivity and specificity, particularly at early stages. In the following section, a general discussion on the application of optical spectroscopic methods as an alternate/adjunct diagnostic tool for oral cancer is presented.

\section{Optical spectroscopy in oral cancer diagnosis}

Spectroscopy is the study of the frequency dependence of the interaction of electromagnetic radiation (light) with matter. Generally, light interacts with matter through absorption, emission and scattering/reflection. In each case, the spectrum of the interaction gives information about, and is characteristic of, the structure and chemical content of the sample. Optical measurements provide quantitative information based on the spectroscopic signature of the biochemical constituents of the sample that can be rapidly analyzed to yield an objective diagnosis, even in the hands of a non-expert operator. Diagnosis is based on biochemical changes underlying the pathology rather than visual or 
microscopic changes in cellular or tissue morphology. Devices to make these measurements have become inexpensive, robust, and portable, because of advances in computing, optical, fiber-optical, and semiconductor technology. Approaches based on fluorescence, Fourier-transform infrared absorption and Raman scattering spectroscopy have shown potential for improved detection of oral cancers. A brief introduction of these techniques and their potential applications in oral cancer diagnosis is presented in the following sub-sections. Figure 2 provides a schematic illustration of their typical method of application.

Fluorescence spectroscopy: When a molecule is illuminated at an excitation wavelength lying within the absorption spectrum of that molecule, it absorbs the energy and undergoes a transition from the ground state to an excited state. The molecule can then relax back from the excited state to the ground state by emission of light at specific emission wavelengths. In the UV/visible/Near infrared region of the spectrum ( 200-1000nm), light emission takes the form of fluorescence (or occasionally phosphorescence). A fluorescence emission spectrum represents the fluorescence intensity measured over a range of emission wavelengths at a fixed excitation wavelength and can provide information relating to the molecular characteristics of the fluorophore, Figure 3.

In the late 1970s, cancer diagnosis based on auto-fluorescence (also called natural, intrinsic or endogenous fluorescence) of naturally occurring fluorophores such as collagen, elastin, keratin and NADH was initiated ${ }^{18}$. The presence of disease can lead to changes in blood concentration, nuclear size, collagen content or epithelial thickness, which can alter the concentration and characteristics of the fluorophores. In oral cancers, it was demonstrated that the epithelial layer shields the strongly fluorescing collagen layer leading to a low intensity of fluorescence in cancers ${ }^{19}$. An ex vivo study used hamster buccal pouch as an experimental model to identify spectral markers associated with different stages of oral carcinogenesis ${ }^{20}$. Onizawa et al., compared fluorescence spectra from human and hamster biopsies and oral cancer cell lines, suggesting that variation in the riboflavin and porphyrin fluorescence can be used as a spectroscopic marker for normal and cancerous conditions ${ }^{21}$. Ingrams et al. further showed that normal and cancerous human biopsies can be discriminated based on their autofluorescence spectral profile ${ }^{22}$. Another ex vivo study by Muller et al., explored the feasibility of quantifying the spectroscopic response of different grades of malignancy ${ }^{23}$. 
The first in vivo study using autofluorescence spectroscopy by Harris et al. reported differences between healthy and tumor mucosa based on the porphyrin emission band ${ }^{24}$. These differences were attributed to microorganisms living on ulcerating or necrotic surfaces. In vivo methods have also been explored to understand oral cancer progression in animal models ${ }^{25}$. Gillenwater et al. recorded in vivo autofluorescence from oral mucosa of 8 healthy volunteers and 15 patients with premalignant or malignant lesions ${ }^{26}$. Decreased intensity in the blue spectral regions, and increased porphyrin fluorescence in the red were observed. Based on the ratio between these, a sensitivity of $82 \%$ and specificity of $100 \%$ were reported ${ }^{26}$. Various other studies have provided further evidence in support of in vivo fluorescence spectroscopy for non-invasive oral cancer diagnosis ${ }^{27-29}$. A recent study by Shaizu et al. showed that autofluorescence spectroscopy can be used to identify oral cavity disorders caused by long-term tobacco habits. Their findings suggest that lower collagen levels and increased ratios of flavin adenine dinucleotide (FAD) to nicotinamide adenine dinucleotide (NADH) can serve as prognostic markers for oral cancer risk ${ }^{30}$.

However, tissue contains few natural fluorophores and their spectroscopic features are broad and overlapping, making them poorly distinguishable, reducing the specificity of fluorescence spectroscopy for diagnostic applications.

Fourier-transform infrared spectroscopy: Vibrational Spectroscopy is a subset of spectroscopy which analyses vibrations within a molecule (or material). The vibrations are characteristic of the molecular structure and, in polyatomic molecules, give rise to a spectroscopic "fingerprint". The spectrum of vibrational energies or frequencies (expressed as wavenumbers, $\mathrm{cm}^{-1}$ ) can thus be employed to characterise a molecular structure, or changes to it due to the local environment or external factors (radiation, chemical agents). Vibrational energies fall within the mid Infrared (IR) region of the electromagnetic spectrum and are commonly probed through IR absorption spectroscopy. High energy or frequency vibrations are characteristic of light, tightly bound groups such as $\mathrm{C}-\mathrm{H}, \mathrm{N}-\mathrm{H}$ and $\mathrm{O}-\mathrm{H}$, whereas low frequencies are associated with heavier groups, or collective vibrations such as ring breathing or skeletal stretches in macromolecules (Figure 4).

IR spectroscopy is now a routine technique for materials characterisation and has found numerous applications in forensics, environmental science and pharmacology ${ }^{31}$. Applications to tissue samples 
for (cancer) diagnostic applications were first reported in the early 90s, and since this time a range of pathologies has been investigated ${ }^{32}$.

Wu et al. demonstrated that, on the basis of lipid and protein content, normal and tumor oral tissues can be discriminated ${ }^{33}$. In another study, of 10 normal sub-gingival tissues (NST) and 15 oral squamous cell carcinoma (SCC) tissues, Fukuyama et al. demonstrated that the normal spectra are strongly influenced by the presence of collagen. They also suggested that spectra are influenced by keratin, which exists in the ectodermal cells ${ }^{34}$. Study using FTIR spectroscopy to understand oral carcinogenesis in animal models have also been reported ${ }^{35}$. FTIR imaging methods have also been explored to analyze different aspects of oral cancers. A study by Schultz et al., to assess changes in biochemistry of well and poorly differentiated oral/oropharyngeal SCC by infrared microspectroscopy, demonstrated that DNA and keratin can provide spectral markers to differentiate between normal and SCC biopsies ${ }^{36}$. Bruni et al., by generating three-dimensional IR chemical maps, demonstrated that proliferating and regressive states of head and neck tumours can be identified ${ }^{37}$. Towards high throughput, automated analysis, Pallua et al. demonstrated that good quality FTIR images can be obtained from formalin fixed paraffin embedded tissue microarray sections providing molecular level information as the basis for diagnosis ${ }^{38}$.

Compared to fluorescence, FTIR provides a detailed fingerprint of the biochemical content of the sample. However, although FTIR has been used for the analysis of human tissues ex vivo, the application of this method for in vivo diagnosis is limited, due to the short penetration depth and the fact that water is highly absorptive in the mid-IR range. Conventional optical fibres have limited transparency in the IR region, and therefore, in vivo studies are less frequent than fluorescence or Raman fibre optical applications. New developments based on attenuated total reflection (ATR) elements might help in implementing in vivo applications.

Raman spectroscopy: Raman spectroscopy is a complementary technique to FTIR and has its origin in the discovery of the Raman effect in 1928, for which C.V. Raman was awarded the Nobel prize in $1930^{39}$. Similar to IR spectroscopy, Raman entails the coupling of incident radiation with molecular vibrations and the resultant spectrum is similarly characteristic of the material. However, whereas IR spectroscopy involves the absorption of radiation, Raman spectroscopy is a scattering technique, 
whereby the incident radiation couples with the vibrating polarisation of the molecule and thus generates or annihilates a vibration. The differing underlying mechanisms results in a complementarity of the techniques. Vibrations of asymmetric, polar bonds thus tend to be strong in IR spectra, whereas Raman is particularly suitable as a probe of symmetric, nonpolar groups. Notably, O$\mathrm{H}$ vibrations of water are very strong in IR spectra, but are extremely weak in Raman spectra, rendering Raman a potentially more suitable technique for in vivo applications (Figure 3). A further implication of the differing physical origins of the techniques is that, whereas IR monitors the absorption of IR radiation, Raman scattering can be employed in the UV, visible or near IR regions of the spectrum (Figure 2). Raman scattering thus offers intrinsically higher spatial resolution for mapping or profiling in a confocal microscopy mode, the limit of spatial resolution being determined by the wavelength $(<1 \mu \mathrm{m}$ for Raman, $\sim 5-10 \mu \mathrm{m}$ for IR). The application of Raman spectroscopy to biomolecules was first demonstrated as early as the 1960s and by the 1970s biomedical applications were explored ${ }^{40}$. Whole cell, tissue and in vivo studies carried out on a range of pathologies have demonstrated the potential for diagnostic applications ${ }^{41}$.

Raman spectroscopic applications in oral cancer diagnostics started with the analysis of normal and dysplastic tissue in a rat model by Schut et al. (2000). Dysplasia in the palate was induced by topical application of the carcinogen 4-nitroquinoline 1-oxide and sensitivity and specificity of $100 \%$ were observed $^{42}$. This was followed by a study of human oral cancer biopsies by Venkatakrishna et al., they recorded spectra of 49 biopsies and obtained an average classification efficiency of $88 \%{ }^{43}$. In 2004 , a study carried out by Krishna et al. demonstrated the applicability of formalin fixed oral tissues for optical pathology, revealing significant differences in the epithelial region of normal and malignant samples, arising from the protein composition, conformational/structural changes, and possible increase in protein content in malignant epithelia ${ }^{44}$. In 2006, Malini et al. demonstrated the efficacy of Raman spectroscopic methods in discriminating normal, cancerous, precancerous and inflammatory conditions $^{45}$. Lipid rich features in normal conditions and prominent protein features in tumors and other pathological conditions were observed. Classification between different groups using multivariate statistical methods produced $100 \%$ sensitivity and specificity ${ }^{45}$. Raman mapping of tissue sections further elucidated biochemical changes within different epithelial layers which are associated 
with disease onset ${ }^{46}$. A study by Sunder et al. demonstrated that oral carcinomas of different pathological grades can also be differentiated on the basis of the relative intensities of bands associated with lipids and proteins ${ }^{47}$.

In vivo Raman spectroscopy using fibreoptic probes for identifying site specific variations in the oral cavity was reported by Guze et al. in 2009, indicating that different oral sites can be discriminated on the basis of level of keratinization ${ }^{48}$. Bergholt et al., (2011), characterized the Raman spectroscopic profiles of different oral cavity regions (inner lip, attached gingiva, floor, dorsal tongue, ventral tongue, hard palate, soft palate, and buccal mucosa ${ }^{49}$. Fitting of reference biochemicals (hydroxyapatite, keratin, collagen, DNA, and oleic acid) and partial least squares-discriminant analysis (PLS-DA) were employed to assess the inter-anatomical variability. The findings suggest that histological and morphological characteristics of different sites have a significant influence on the in vivo Raman spectra, and different sites can be classified with an overall sensitivity and specificity of $85 \%{ }^{49}$. Singh et al. demonstrated that in vivo spectra can be acquired in clinically implementable timescales and demonstrated the feasibility of classification of normal and pathological conditions ${ }^{50}$. This was followed by another study exploring tobacco induced cancer field effects in the oral mucosa $^{51}$. Sahu et al. demonstrated that in vivo Raman spectroscopy methods can also be utilized to understand age-related changes in the oral mucosa ${ }^{52}$. These findings were further verified by a recent study showing anatomical variability and feasibility of identifying pathological conditions with in vivo Raman spectroscopy ${ }^{53}$.

Minimally invasive methods in oral cancer diagnosis: Bio fluids such as blood, urine, lymph, and saliva can provide substantial information about human health and are being widely investigated for clinical diagnosis of various diseases including oral cancers. The attraction of these specimens lies in the fact that they can be used for mass screening, due to ease in collection, transport and low cost ${ }^{54}$. Studies have been carried out on physiochemical properties of saliva using surface enhanced laser desorption and ionization time of flight (SELDI-TOF) coupled with mass spectrometry (MS) and high performance liquid chromatography (HPLC) to identify proteomic and enzymatic markers associated with oral cancer $^{55}$. Other techniques such as laser-induced fluorescence coupled with HPLC, and 
capillary electrophoresis coupled mass spectroscopy have been employed to characterise salivary metabolites in oral cancer patients ${ }^{56,57}$.

Recently, optical methods based on Raman, infrared absorption, and fluorescence spectroscopies have also been exploited for such investigations. For example, enhanced levels of porphyrin in blood have been used as a diagnostic marker for various cancers including oral cancers $^{58,59}$. Yuvaraj et al. characterized different salivary metabolites associated with oral cancers by fluorescence spectroscopy ${ }^{60}$. FTIR spectroscopy has been applied to study sputum in order to diagnose oral cancers and discrimination between normal and cancerous samples was achieved on the basis of changes in the protein and glycoprotein structure within cells ${ }^{61}$. Surface-Enhanced Raman spectroscopy methods (SERS) have been used to differentiate between normal and oral cancer patients using spectra acquired from saliva ${ }^{62}$. A recent study by Elumalai et al. demonstrated that Raman spectroscopy of urine samples of healthy subjects and oral cancer patients can offer potential diagnostic information with a discrimination accuracy of $94 \%{ }^{63}$. The analysis of exfoliated oral cells by optical methods also holds enormous promise for early disease detection and diagnosis. Diem and co-workers have carried out multiple studies on spectral cytopathology of oral exfoliated cells ${ }^{64,65}$. Their findings are suggestive of the tremendous potential of spectroscopic methods in identifying minor changes associated with disease onset. Nevertheless, diagnosis based on biofluids suffers from limitations such as low analyte concentration, longer acquisition time, prone to experimental errors etc. Considerable efforts have been undertaken to develop standard protocols and sensitive instrumentation. Signal enhancements with the help of nano-particles or surface coating is an active area of research ${ }^{66}$. Concentration of samples using centrifugal filtration devices has been shown to offer an alternative which allows measurement of the analytes in the native aqueous environment ${ }^{67}$. This also allows fractionation according to molecular weight of the constituent analytes, potentially allowing the targeting of molecular biomarkers of a disease. Appropriate modification in the instrumentation, especially automation for collection and analysis of body fluids is also an area which requires constant development. Efforts should also be undertaken for large scale trials and database development to overcome inter-laboratory and instrument variabilities ${ }^{68}$. 


\section{Summary and Outlook}

Although the oral cavity is easily accessible to inspection, oral cancer patients often present at an advanced stage when treatment is less successful, thereby leading to high morbidity and mortality. Early detection is the best way to ensure patient survival and quality of life. The current gold standard for clinical diagnosis of oral lesions is biopsy and subsequent histopathological confirmation. The process is invasive, time-consuming and prone to inter-observer variability. An alternate method of diagnosis that can enable non-invasive diagnosis of the oral cavity in individuals with suspicious oral lesions is warranted.

It is now well recognized that techniques based on optical spectroscopy can play a very important role towards this end. Spectroscopic measurements of tissue biochemistry, with sensitivity and specificity to localize changes enhanced by imaging, represent a measure of health (or disease) unattainable in current practice, and can provide sensitivities for early stage detection of biochemical, rather than simply morphological, abnormalities. Table 1 lists the advantages and disadvantages of the current screening/diagnostic methods and optical spectroscopy methods. Among the spectroscopic techniques described, fluorescence is perhaps the most technologically accessible, as it is simply based on the analysis of light which is emitted after illumination with a UV lamp. The emitted light is in the visible range and therefore probes can use free space or low grade, inexpensive fibre optics. The technique detects only the small fraction of endogenous biomolecules which are fluorescent, however, and relies on identifying pathology specific biomarkers amongst them. FTIR spectroscopy, on the other hand, produces a label free fingerprint of the complete biochemical content of the tissue, cell or biofluid, and this can explore more global and specific pathological changes. However, water is an extremely strong FTIR absorber, and so in vivo diagnostic applications may be limited. Raman spectroscopy provides a similar complete, label free fingerprint of the sample, and also couples to benefits of working in the visible region of the spectrum. Water is a weak Raman scatterer, and so the technique is more adaptable to routine in vivo patient screening or ex vivo spectral histology or cytology. The prospect is therefore of a high sensitivity and specificity, automatable, objective quantitative label free probe of early stage disease development and progression, based on the biomolecular content of the patient sample. 
Variable thickness and degree of keratinisation at different sites in the oral cavity can influence the diagnostic efficacy of optical methods, especially for early lesions. This issue has been addressed extensively by the biomedical spectroscopic community. Various studies have successfully demonstrated the potential of spectroscopic methods in identifying anatomical variability due to different levels of keratinization ${ }^{19,28,29,48,49}$. These studies have provided evidence in support of inherent differences between different locations and suggested that each site be treated independently. For example, spectral models developed using spectra from buccal mucosa cancers cannot be used for identifying abnormalities at tongue or palate. Most of the recent studies have been performed under these guidelines, where tumors of specific sites are treated separately ${ }^{50-53}$. As with all optical techniques, the depth sensitivity is limited by the absorption and scattering of the tissue. Operation in the near infrared can optimise the depth sensitivity of Raman probes, and novel methodologies such as spatially offset Raman spectroscopy (SORS) promise increased penetration depths of several millimetres for deeper set lesions ${ }^{69}$. Such technological advances potentially place Raman ahead of the field as candidate for in vivo optical diagnostic applications.

In the coming years, large scale clinical trials must be conducted to gain the amount of sitespecific data necessary for developing adequate size training and test sets for robust algorithm development and analysis. The standard models for each of the individual sites in the oral cavity should be tested rigorously, preferably double-blinded, as multi-centric studies, before they are considered for routine use. Several technological advances in terms of fiberoptic probes and miniaturization of instruments are also required for real time and routine diagnosis. Efficient suppression of background signal, optimization of collection optics, and incorporation of miniaturized interference filters in the fiber probes are some of the issues that are to be addressed effectively. Further improvements in data analysis algorithms are also required for developing less cumbersome, rapid, unambiguous, objective and user friendly interfaces from the point of view of routine clinical use where a clinician or a technician can analyze a given spectrum against all available models to diagnose a case. The prospective adaptation of optical spectroscopy methods for routine clinical diagnosis would decrease the number of follow-up clinic visits and patient anxiety by minimizing 
waiting times for histopathological diagnosis. The technology poses no known risks to the patients, and therefore could be a safe alternative/adjunct to the current diagnostic methods. 


\section{References}

1. Ferlay J, Soerjomataram I, Dikshit R, Eser S, Mathers C, Rebelo M et al. Cancer incidence and mortality worldwide: Sources, methods and major patterns in GLOBOCAN 2012. Int J Cancer 2014;136:359-386.

2. Warnakulasuriya S. Global epidemiology of oral and oropharyngeal cancer. Oral Oncol 2009;45:309-316.

3. Pindborg J, Reichart P, Smith C, Van der Waal I. World Health Organization International Histological Classification of Tumours. Histological typing of cancer and precancer of the oral mucosa. Berlin: Springer; 1997.

4. Silverman S, Jr., Gorsky M, Lozada F. Oral leukoplakia and malignant transformation. A follow-up study of 257 patients. Cancer 1984;53: 563-8.

5. Lumerman H, Freedman P, Kerpel S. Oral epithelial dysplasia and the development of invasive squamous cell carcinoma. Oral Surg Oral Med Oral Pathol Oral Radiol Endod 1995;79: 3219.

6. Murti PR, Bhonsle RB, Pindborg JJ, Daftary DK, Gupta PC, Mehta FS. Malignant transformation rate in oral submucous fibrosis over a 17-year period. Community Dent Oral Epidemiol 1985;13: 340-1.

7. Silverman S, Jr., Gorsky M, Lozada-Nur F. A prospective follow-up study of 570 patients with oral lichen planus: persistence, remission, and malignant association. Oral Surg Oral Med Oral Pathol 1985;60: 30-4.

8. Blot WJ, McLaughlin JK, Winn DM, Austin DF, Greenberg RS, Preston-Martin S et al. Smoking and drinking in relation to oral and pharyngeal cancer. Cancer Res 1988;48: 3282-7.

9. Pfeifer GP, Denissenko MF, Olivier M, Tretyakova N, Hecht SS, Hainaut P. Tobacco smoke carcinogens, DNA damage and p53 mutations in smoking-associated cancers. Oncogene 2002;21: 7435-51.

10. Boffetta P, Hashibe M. Alcohol and cancer. Lancet Oncol. 2006;7:149-156 
11. Boffetta P, Hecht S, Gray N, Gupta P, Straif K. Smokeless tobacco and cancer. Lancet Oncol 2008;9: 667-75.

12. Kreimer AR, Clifford GM, Boyle P, Franceschi S. Human papillomavirus types in head and neck squamous cell carcinomas worldwide: a systematic review. Cancer Epidemiol Biomarkers Prev 2005;14: 467-75.

13. Sankaranarayanan R, Ramadas K, Thomas G, Muwonge R, Thara S, Mathew B, Rajan B, Trivandrum Oral Cancer Screening Study G. Effect of screening on oral cancer mortality in Kerala, India: a cluster-randomised controlled trial. Lancet 2005;365: 1927-33.

14. Gandolfo S, Pentenero M, Broccoletti R, Pagano M, Carrozzo M, Scully C. Toluidine blue uptake in potentially malignant oral lesions in vivo: clinical and histological assessment. Oral oncol 2006;42: 89-95.

15. Fist S. The oral brush biopsy: separating fact from fiction. Oral Surg Oral Med Oral Pathol Oral Radiol Endod 2003;96: 654-5.

16. Eisen D, Frist S. The relevance of the high positive predictive value of the oral brush biopsy. Oral oncol 2005;41: 753-5.

17. Abbey LM, Kaugars GE, Gunsolley JC, Burns JC, Page DG, Svirsky JA et al. Intraexaminer and interexaminer reliability in the diagnosis of oral epithelial dysplasia. Oral Surg Oral Med Oral Pathol Oral Radiol Endod 1995;80: 188-91.

18. Profio AE, Doiron DR. A feasibility study of the use of fluorescence bronchoscopy for localization of small lung tumours. Phys Med Biol 1977;22: 949-57.

19. Kolli VR, Savage HE, Yao TJ, Schantz SP. Native cellular fluorescence of neoplastic upper aerodigestive mucosa. Arch Otolaryngol Head Neck Surg 1995;121: 1287-92.

20. Farwell DG, Meier JD, Park J, Sun Y, Coffman H, Poirier B et al. Time-resolved fluorescence spectroscopy as a diagnostic technique of oral carcinoma: Validation in the hamster buccal pouch model. Arch Otolaryngol Head Neck Surg 2010;136: 126-33.

21. Onizawa K, Okamura N, Saginoya H, Yusa H, Yanagawa T, Yoshida H. Analysis of fluorescence in oral squamous cell carcinoma. Oral oncol 2002;38: 343-48. 
22. Ingrams DR, Dhingra JK, Roy K, Perrault DF, Jr., Bottrill ID, Kabani S et al. Autofluorescence characteristics of oral mucosa. Head Neck 1997;19: 27-32.

23. Muller MG, Valdez TA, Georgakoudi I, Backman V, Fuentes C, Kabani S et al. Spectroscopic detection and evaluation of morphologic and biochemical changes in early human oral carcinoma. Cancer 2003;97: 1681-92.

24. Harris DM, Werkhaven J. Endogenous porphyrin fluorescence in tumors. Lasers Surg Med 1987; 7: 467-72.

25. Wang CY, Tsai T, Chen HC, Chang SC, Chen CT, Chiang CP. Autofluorescence spectroscopy for in vivo diagnosis of DMBA-induced hamster buccal pouch pre-cancers and cancers. J Oral Pathol Med 2003;32: 18-24.

26. Gillenwater A, Jacob R, Ganeshappa R, Kemp B, El-Naggar AK, Palmer JL et al. Noninvasive diagnosis of oral neoplasia based on fluorescence spectroscopy and native tissue autofluorescence. Arch Otolaryngol Head Neck Surg 1998;124: 1251-8.

27. Chaturvedi P, Majumder SK, Krishna H, Muttagi S, Gupta PK. Fluorescence spectroscopy for noninvasive early diagnosis of oral mucosal malignant and potentially malignant lesions. J Cancer Res Ther 2010;6: 497-502.

28. Mallia RJ, Subhash N, Sebastian P, Kumar R, Thomas SS, Mathews A, Madhavan J. In vivo temporal evolution of ALA-induced normalized fluorescence at different anatomical locations of oral cavity: application to improve cancer diagnostic contrast and potential. Photodiagnosis Photodyn Ther 2010;7: 162-75.

29. Schwarz RA, Gao W, Daye D, Williams MD, Richards-Kortum R, Gillenwater AM. Autofluorescence and diffuse reflectance spectroscopy of oral epithelial tissue using a depth-sensitive fiber-optic probe. Appl opt 2008;47: 825-34.

30. Nazeer Shaiju S, Ariya S, Asish R, Salim Haris P, Anita B, Arun Kumar G et al. Habits with killer instincts: in vivo analysis on the severity of oral mucosal alterations using autofluorescence spectroscopy. J biomed opt 2011;16: 087006.

31. Messerchmidt R., Harthcock M. (1988). Infrared Microscopy, Theory and Applications, New York, Marcel Dekker 
32. Dukor RK. Vibrational Spectroscopy in the Detection of Cancer Handbook of Vibrational Spectroscopyed.: John Wiley \& Sons, Ltd, 2006.

33. Wu JG, Xu YZ, Sun CW, Soloway RD, Xu DF, Wu QG et al. Distinguishing malignant from normal oral tissues using FTIR fiber-optic techniques. Biopolymers 2001;62: 185-92.

34. Fukuyama Y, Yoshida S, Yanagisawa S, Shimizu M. A study on the differences between oral squamous cell carcinomas and normal oral mucosas measured by Fourier transform infrared spectroscopy. Biospectroscopy 1999;5: 117-26.

35. Krishnakumar N, Sulfikkarali NK, Manoharan S, Nirmal RM. Screening of chemopreventive effect of naringenin-loaded nanoparticles in DMBA-induced hamster buccal pouch carcinogenesis by FT-IR spectroscopy. Mol Cell Biochem 2013;382: 27-36.

36. Schultz CP, Liu KZ, Kerr PD, Mantsch HH. In situ infrared histopathology of keratinization in human oral/oropharyngeal squamous cell carcinoma. Oncol Res 1998;10: 277-86.

37. Bruni P, Conti C, Giorgini E, Pisani M, Rubini C, Tosi G. Histological and microscopy FT-IR imaging study on the proliferative activity and angiogenesis in head and neck tumours. Faraday discuss 2004;126: 19-26; discussion 77-92.

38. Pallua JD, Pezzei C, Zelger B, Schaefer G, Bittner LK, Huck-Pezzei VA et al. Fourier transform infrared imaging analysis in discrimination studies of squamous cell carcinoma. Analyst 2012;137: 3965-74.

39. Raman C.V. and Krishnan K.S. A change of wavelength in light scattering. Nature 1928: $121,501$.

40. Walton AG, Deveney MJ, Koenig JL. Raman spectroscopy of calcified tissue. Calcif Tissue Res 1970;6: 162-7.

41. Hanlon EB, Manoharan R, Koo TW, Shafer KE, Motz JT, Fitzmaurice M et al. Prospects for in vivo Raman spectroscopy. Phys Med Biol 2000;45: R1-59.

42. Bakker Schut TC, Witjes MJ, Sterenborg HJ, Speelman OC, Roodenburg JL, Marple ET et al. In vivo detection of dysplastic tissue by Raman spectroscopy. Anal chem 2000;72: 6010-8. 
43. Venkatakrishna K, Kurien J, Keerthilata MP, Valiathan M, Kumar NN, Krishna CM et al. Optical pathology of oral tissue: A Raman Spectroscopy diagnostic method. Current Science 2001;80:101-105.

44. Krishna CM, Sockalingum GD, Kurien J, Rao L, Venteo L, Pluot M et al. Micro-Raman spectroscopy for optical pathology of oral squamous cell carcinoma. Appl Spectrosc 2004;58: 112835.

45. Malini R, Venkatakrishna K, Kurien J, Pai KM, Rao L, Kartha VB et al. Discrimination of normal, inflammatory, premalignant, and malignant oral tissue: a Raman spectroscopy study. Biopolymers 2006;81: 179-93.

46. Cals FLJ, Bakker Schut TC, Koljenović S, Puppels GJ, de Jong RJB. Method development: Raman spectroscopy-based histopathology of oral mucosa. J Raman Spectrosc 2013;44: 963-72.

47. Sunder NS, Rao Nirmala N, Kartha VB, Ullas G, Kurien J. Laser Raman spectroscopy: A novel diagnostic tool for oral cancer. J Orofac Sci.2011;3:15-19.

48. Guze K, Short M, Sonis S, Karimbux N, Chan J, Zeng H. Parameters defining the potential applicability of Raman spectroscopy as a diagnostic tool for oral disease. J Biomed Opt 2009;14: 014016.

49. Bergholt MS, Zheng W, Huang Z, Characterizing variability in in vivo Raman spectroscopic properties of different anatomical sites of normal tissue in the oral cavity. $\mathrm{J}$ Raman Spectrosc 2012;43:255-262.

50. Singh SP, Deshmukh A, Chaturvedi P, Krishna CM. In vivo Raman spectroscopic identification of premalignant lesions in oral buccal mucosa. J Biomed Opt 2012;17: 105002.

51. Singh SP, Sahu A, Deshmukh A, Chaturvedi P, Krishna CM. In vivo Raman spectroscopy of oral buccal mucosa: a study on malignancy associated changes (MAC)/cancer field effects (CFE). Analyst 2013;138: 4175-82.

52. Sahu A, Deshmukh A, Ghanate AD, Singh SP, Chaturvedi P, Krishna CM. Raman spectroscopy of oral buccal mucosa: a study on age-related physiological changes and tobacco-related pathological changes. Technol Cancer Res Treat 2012;11: 529-41. 
53. Krishna H, Majumder SK, Chaturvedi P, Gupta PK. Anatomical variability of in vivo Raman spectra of normal oral cavity and its effect on oral tissue classification. Biomed Spectrosc Imaging 2013;2: 199-217.

54. Rajasekaran R, Aruna PR, Koteeswaran D, Padmanabhan L, Muthuvelu K, Rai RR et al. Characterization and diagnosis of cancer by native fluorescence spectroscopy of human urine. Photochem Photobiol 2013;89: 483-91.

55. Bigler LR, Streckfus CF, Dubinsky WP. Salivary biomarkers for the detection of malignant tumors that are remote from the oral cavity. Clin. Lab. Med. 2009;29: 71-85.

56. Sugimoto M, Wong DT, Hirayama A, Soga T, Tomita M. Capillary electrophoresis mass spectrometry-based saliva metabolomics identified oral, breast and pancreatic cancer-specific profiles. Metabolomics 2010;6: 78-95.

57. Venkatakrishna K, Kartha VB, Pai KM, Krishna CM, Ravikiran O, Kurian J et al. HPLCLIF for early detection of oral cancer. Current Science 2003;84: 551-57.

58. Meng JW, Wang XJ, Ma HP, Ren XG, Xu XR, Ren WM. Protoporphyrin IX fluorescence from the plasma of tumor-implanted mouse. J Lumin 1999;83-84: 251-4.

59. Madhuri S, Vengadesan N, Aruna P, Koteeswaran D, Venkatesan P, Ganesan S. Native fluorescence spectroscopy of blood plasma in the characterization of oral malignancy. Photochem Photobiol 2003;78: 197-204.

60. Yuvaraj M, Udayakumar K, Jayanth V, Prakasa Rao A, Bharanidharan G, Koteeswaran D et al. Fluorescence spectroscopic characterization of salivary metabolites of oral cancer patients. J Photochem Photobiol B 2014;130: 153-60.

61. Menzies GE, Fox HR, Marnane C, Pope L, Prabhu V, Winter S et al. Fourier transform infrared for noninvasive optical diagnosis of oral, oropharyngeal, and laryngeal cancer. Transl Res 2014;163: 19-26.

62. Olivo M, Bhuvaneswari R, Keogh I. Advances in Bio-Optical Imaging for the Diagnosis of Early Oral Cancer. Pharmaceutics 2011;3: 354-78.

63. Elumalai B, Prakasarao A, Ganesan B, Dornadula K, Ganesan S. Raman spectroscopic characterization of urine of normal and oral cancer subjects. J Raman Spectrosc 2015;46:84-93. 
64. Papamarkakis K, Bird B, Schubert JM, Miljkovic M, Wein R, Bedrossian K et al. Cytopathology by optical methods: spectral cytopathology of the oral mucosa. Lab Invest 2010;90: $589-98$.

65. Diem M, Mazur A, Lenau K, Schubert J, Bird B, Miljkovic M et al. Molecular pathology via IR and Raman spectral imaging. J Biophotonics 2013;6: 855-86.

66. Kah JCY, Kho KW, Lee CGL, Richard CJ, Sheppard, Shen ZX et al. Early diagnosis of oral cancer based on the surface plasmon resonance of gold nanoparticles. Int $\mathbf{J}$ Nanomedicine 2007;2: 785-98.

67. Bonnier F, Baker MJ, Byrne HJ. Vibrational spectroscopic analysis of body fluids: avoiding molecular contamination using centrifugal filtration. Anal Methods 2014;6:5155

68. Byrne HJ, Baranska M, Puppels GJ, Stone N, Wood B, Gough KM et al. Spectropathology for the Next Generation: Quo Vadis?. Analyst 2015;140:2066-73.

69. Matousek P, Stone N. Recent advances in the development of Raman spectroscopy for deep non-invasive medical diagnosis. J Biophotonics 2013; 6: 7-19. 


\section{Figure legends}

Figure 1: Clinical presentation of tongue- cancer (A), leukoplakia (B) and lichen planus (C)

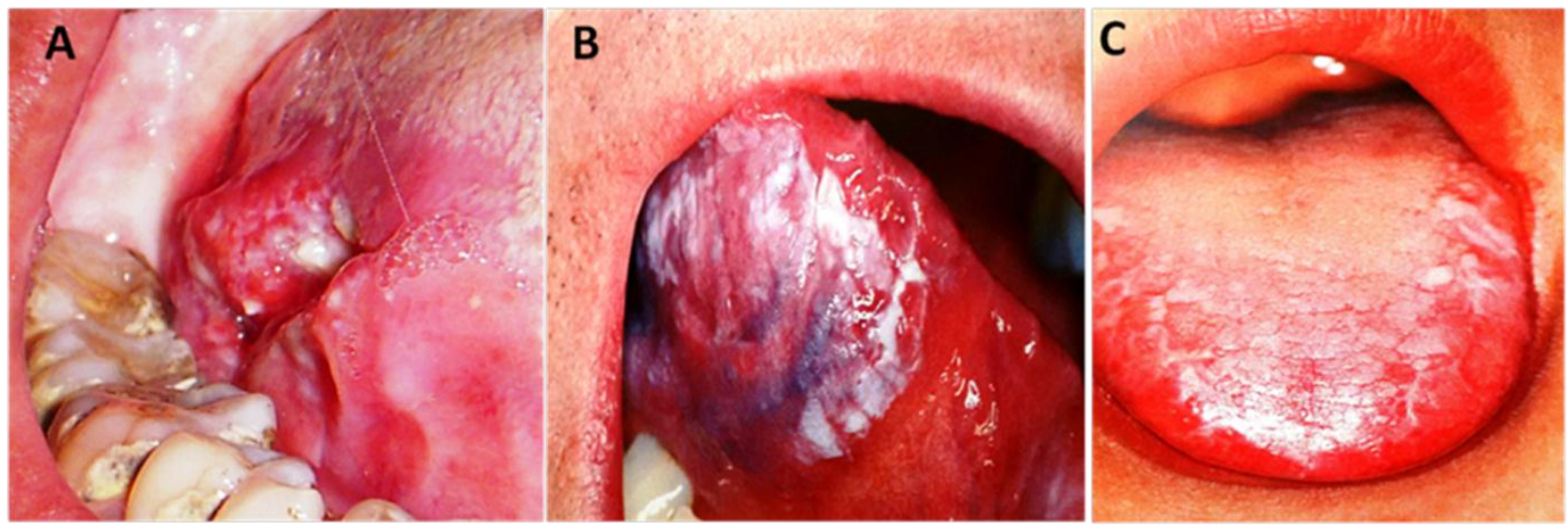


Figure 2: A schematic of the typical application of optical spectroscopic techniques for diagnostic applications. The light source is delivered via a probe or microscope (for in-vivo or ex vivolin-vitro applications respectively) to the sample (cells, tissue or biofluid). Upon excitation by an appropriate source, molecules can either; go to an excited state and reemit light in the form of fluorescence (UV/visible lamp), absorb the light to generate vibrations within the molecules (Infrared lamp), or, by interaction with the vibrational modes of the molecules in the cells; the light is Raman scattered (visible or near infrared laser). The emitted/transmitted/scattered light is then collected by the probe or microscope and passed to a detector. The operator can then perform analysis on the resulting spectra and, using a prepared classifier, the output can for example be a yes, no, or maybe for the presence of cancer. The technique can be modified according to the application; in-vivo, ex-vivo (histological or cytological) or in vitro.

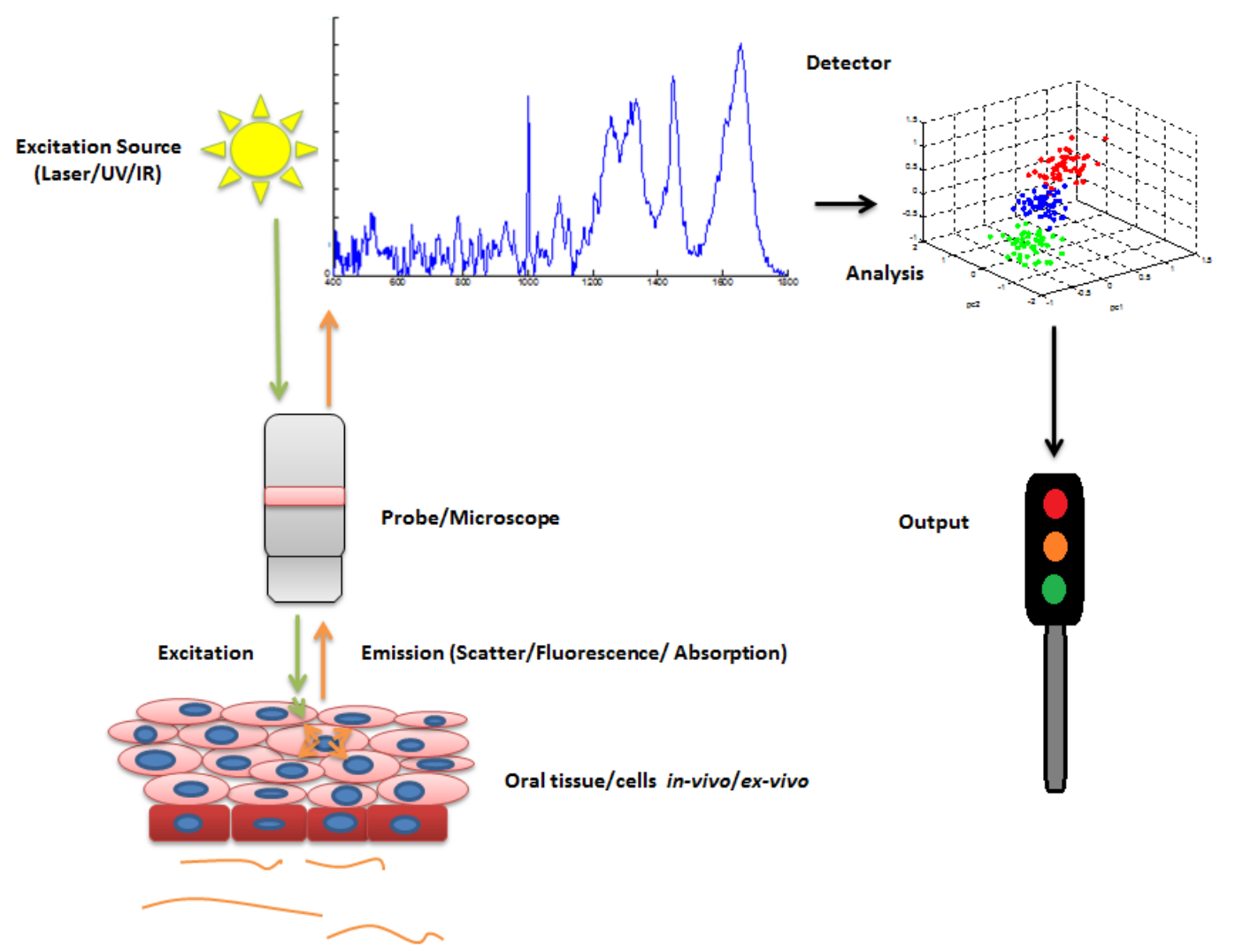


Figure 3: Comparison of typical fluorescence spectrum (riboflavin) and typical Raman spectrum of tissue (both normalised) plotted on an energy scale. The intrinsic bandwidth of the fluorescence feature is very broad, compared to the Raman spectrum, which has a multitude of narrow bands which are shifted from the source wavelength of $785 \mathrm{~nm}(\sim 1.6 \mathrm{eV})$. The energy shift of the Raman band is a measure of the vibrational energy and for comparison with infra red spectroscopy is usually expressed in wavenumbers $(1 / \mathrm{cm})$. Note the energy scales for the two spectra are different.

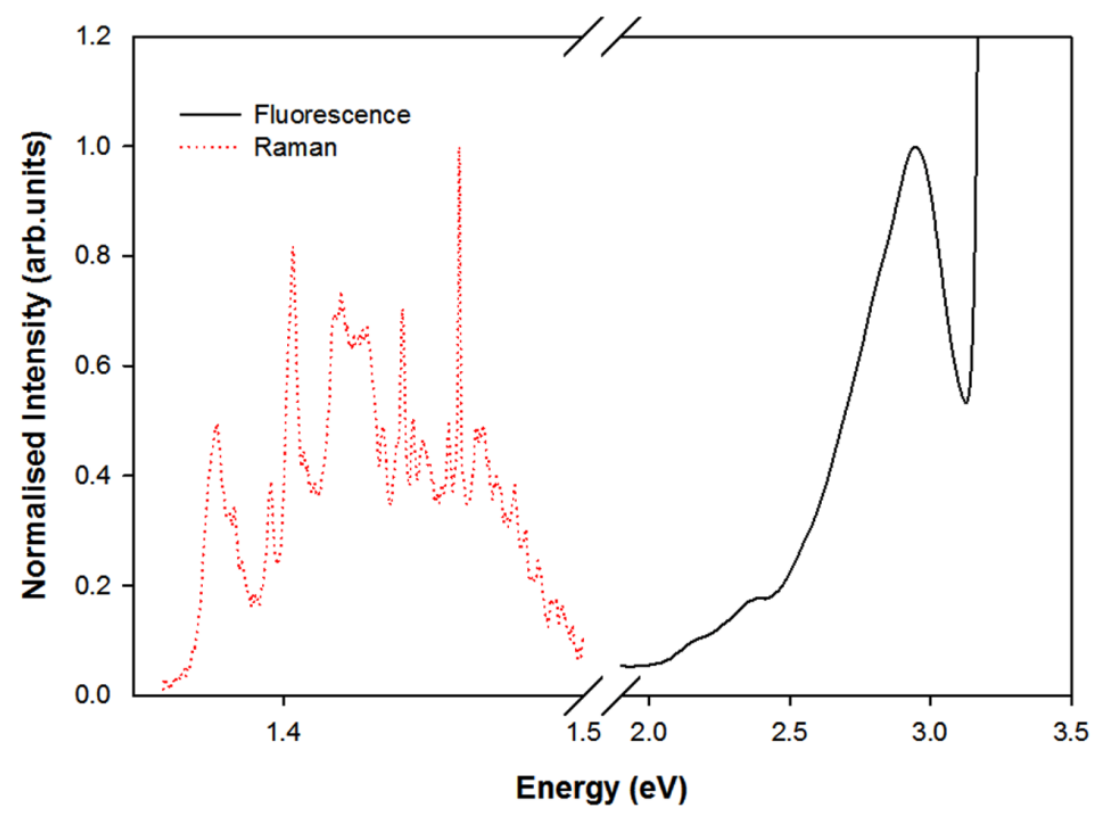


Figure 4: Comparison of typical IR absorption and Raman spectra of human tissue samples. The IR spectrum is in the mid infrared region of the spectrum, and the spectrum is less rich in information than the Raman spectrum. The Raman spectrum is expressed as wavenumber shift from the source laser line, although as shown in Figure 3, the scattered light is in the visible region of the spectrum. Both show typical features of lipids $(\bullet)$, proteins $(\boldsymbol{\Delta})$, carbohydrates $(\boldsymbol{\square})$ and nucleic acids $(\diamond)$. Note the strong absorption due to trace water in the FTIR spectrum in the region of $\sim 3300 \mathrm{~cm}^{-1}$.

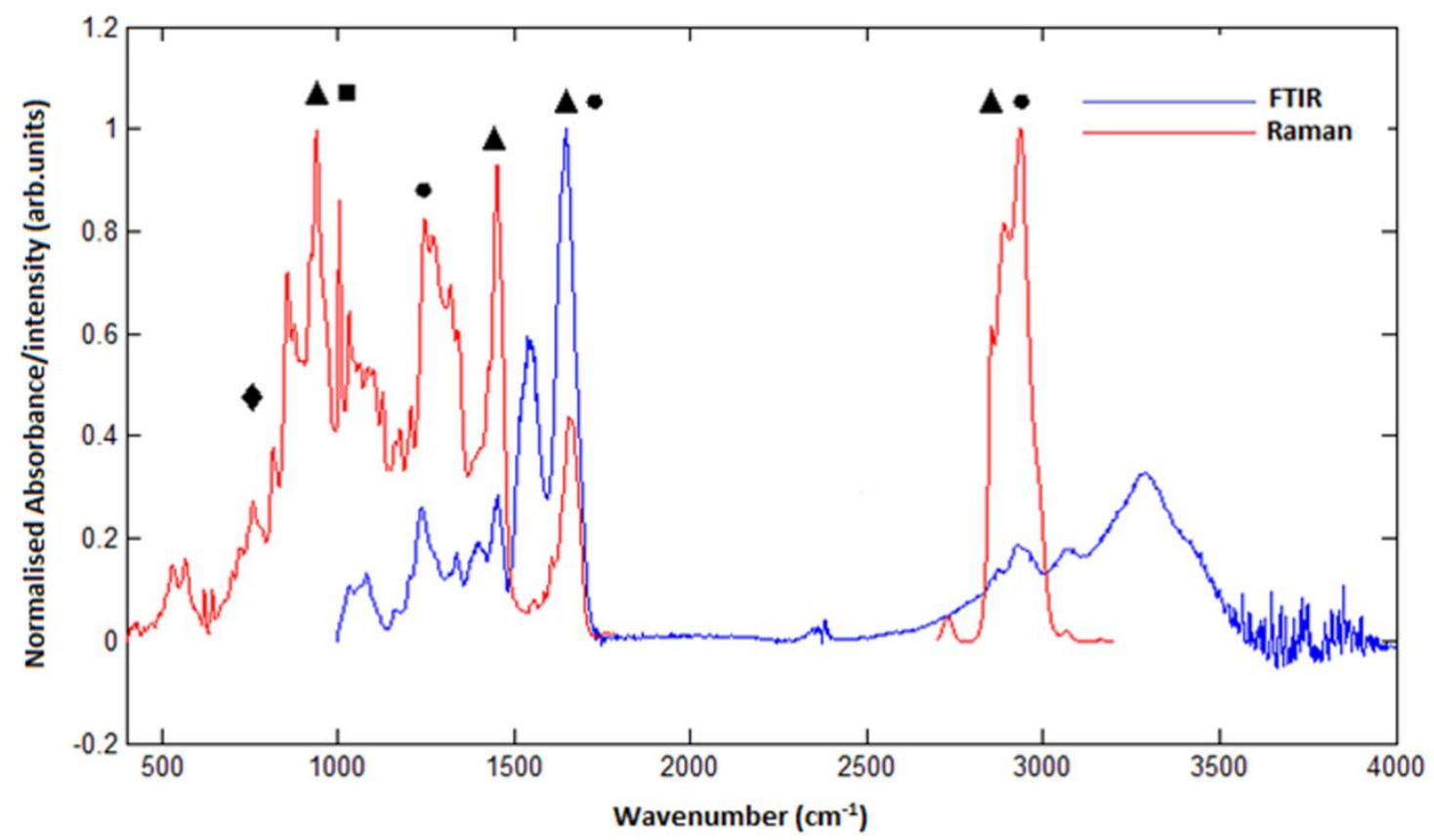

